\title{
A Review of the Application of Deep Learning in Brachytherapy
}

\author{
Hai Hu*, Yang Shao, Shijie Hu \\ Applied Nuclear Technology in Geosciences Key Laboratory of Sichuan Province, Chengdu University of Technology, Chengdu, \\ China \\ Email: ^hh511322@126.com
}

How to cite this paper: $\mathrm{Hu}, \mathrm{H}$., Shao, $\mathrm{Y}$. and $\mathrm{Hu}$, S.J. (2020) A Review of the Application of Deep Learning in Brachytherapy. Open Access Library Journal, 7: e6589. https://doi.org/10.4236/oalib.1106589

Received: July 6, 2020

Accepted: July 25, 2020

Published: July 28, 2020

Copyright (c) 2020 by author(s) and Open Access Library Inc.

This work is licensed under the Creative Commons Attribution International License (CC BY 4.0).

http://creativecommons.org/licenses/by/4.0/ (c) (i) Open Access

\begin{abstract}
Objective The automation of brachytherapy is the direction of future development. This article retrospectively studied the application of deep learning in brachytherapy of cervical cancer and clarified the status quo of development. Method This survey reviewed the application of machine learning and deep learning in brachytherapy for cervical cancer in the past 10 years. The survey retrieved and reviewed electronic journal articles in scientific databases such as Google Scholar and IEEE. The three sets of keywords used 1) deep learning, brachytherapy, 2) machine learning, brachytherapy, 3) automation, brachytherapy. Results Through research on the application of deep learning in brachytherapy, it is found that the U-net model is basically based on convolutional neural networks or some attention mechanisms are added to it, and it is applied to brachytherapy of prostate or cervical cancer. The automatic segmentation and reconstruction of the mid-source applicator (interpolation needle), target area delineation, optimization in the treatment planning system and dose calculation have achieved good results, proving that deep learning can be applied to the clinical treatment of brachytherapy. Conclusion The research on the application of deep learning in brachytherapy confirmed that deep learning can effectively promote the development of brachytherapy.
\end{abstract}

\section{Subject Areas}

Psychiatry \& Psychology

\section{Keywords}

Deep Learning, Brachytherapy, Machine Learning, Automation

\section{Introduction}

Brachytherapy technology is a method of placing a radioactive source into the 
tumor area through an applicator or directly implanting it into the tumor tissue for radiotherapy. Because of its inherent physical and biological characteristics, brachytherapy can give a high absorbed dose to the focused irradiation of the tumor, and the dose around the source drops rapidly, which can effectively increase the local irradiation of the tumor and protect the normal tissue around the tumor. The local control rate and survival rate have significantly reduced the complications associated with brachytherapy. When the patient moves or the tumor moves in the body, the relative position of the radiation source and the tumor can remain unchanged, and the tumor obtains high dose conformity. These advantages make brachytherapy widely used in clinical applications, often used in the cervix and uterus. The treatment of tumors in the body, vagina, nasopharynx, esophagus, rectum, breast, prostate, skin and other parts is also applicable to the treatment of tumors in many other parts [1] [2] [3].

The basic procedures of brachytherapy include: target area delineation, applicator reconstruction, dose calculation and dose optimization [4]. The specific process is shown in Figure 1. This review revolves around the brachytherapy process.

In recent years, with the development of computer hardware, the rapid calculation and innovation of large amounts of data and neural network algorithms brought by social digitization, deep learning technology has developed rapidly, and deep learning has also gradually emerged in the medical field [5] mainly applied in the following aspects: organ delineation, applicator reconstruction, dose calculation and treatment planning system [6] [7] [8]. How to closely integrate emerging deep learning with traditional brachytherapy technology to promote the development of three-dimensional brachytherapy technology is the problem considered in this article, but also provides a broader perspective in this field and finds new problems. This article reviews the application of deep learning in the brachytherapy automation process [9], which is reported as follows.

\section{Organ Delineation and Segmentation}

In brachytherapy, organ delineation and segmentation undoubtedly play an important role in the treatment plan. The patient performs layer-by-layer scanning through CT or MRI to obtain multiple medical images [10]. The doctor sketches the primary target area, the medium-risk target area and the organs at risk according to the patient's condition. In clinical practice, manual sketching is still the main method. However, there are hundreds of CT images of a single patient, which is a lot of work for doctors. The automatic segmentation of the target area of medical images also plays an increasingly important role in helping doctors delineate tumor areas and endanger organs. Many segmentation methods have been developed, and the effects are different according to the type of application and the image studied. Now some of them have mature segmentation methods integrated in commercial treatment planning system [6]. 


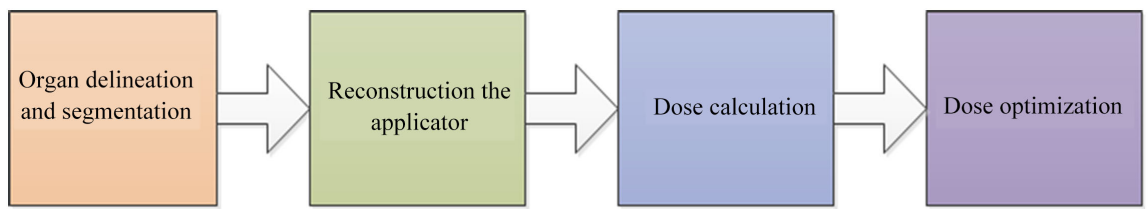

Figure 1. The basic procedures of brachytherapy.

Derek Allman [11] et al. used convolutional neural networks in 2018 to locate and classify the sources and artifacts in the K-wave simulation formation data. The experimental results show that, unlike geometry-based beamforming, the use of convolutional neural networks can be effective to eliminate metal artifacts in the image. In 2018, Xia Huang [12] et al. constructed a convolutional neural network and combined residual learning to eliminate metal artifacts on CT images. The final signal-to-noise ratio on the test set was 38.09. In 2019, Davood Karimi [13] et al. used Convolutional Neural Networks (CNN) to develop an automated accurate and stable segmentation method for the clinical target volume of transrectal ultrasound imaging of the prostate in brachytherapy, and proposed two different strategies to Improving the accuracy of image segmentation, research shows that this method can significantly improve the performance of medical image segmentation. Yang Lei [14] et al. built the supervised learning model V-net in 2019 to complete the segmentation of the prostate in the ultrasound image, and marked the prostate tissue through deep supervised learning. Finally, the segmented prostate volume is reconstructed and refined through contours. Experiments prove that the proposed technique can be used for the diagnosis and treatment of the prostate. The results show that the Days similarity coefficient, Hausdorff distance, and average surface distance of prostate segmentation are $0.92,3.94$, and 0.60 , respectively. Nathan Orlando [15] et al. completed the automatic segmentation of the prostate in ultrasound-guided prostate cancer by constructing U-net and V-net networks in 2020, proving that the constructed model achieved good results in segmentation, and the evaluation parameters all indicated that the segmentation effect was better good. Qin Nannan [16] et al. completed the automatic delineation of clinical target areas and endangered organs in brachytherapy of cervical cancer by building a U-net network in 2020. The average value of the Dess similarity coefficient of the automatically delineated target area is 0.898 , Hausdorff distance. The average value is within $5.3 \mathrm{~mm}$, which proves that it can be used in clinic and can greatly improve the efficiency of doctors.

\section{Segmentation and Reconstruction of the Applicator (Interstitial Needles)}

The applicators serve as a bridge between the radiation source and the patient in brachytherapy, and are extremely important in brachytherapy. Due to the difference in density and patient tissue, highlight features are displayed on the CT image. In the formulation of the treatment plan, it is especially important to re- 
construct the contour of the applicator. The subsequent source distribution and dose optimization are all based on the accurate reconstruction of the applicator. Deep learning and reconstruction of the applicator are also the direction of development at this stage. Nlls Gessert [17] et al. built a deep learning model based on spatial continuity in 2019 to estimate the position of the tip when the interpolation needle is inserted, to solve problems in clinical applications, and proved that this model can also be used to adjust the position of the calibration interpolation needle. The relationship coefficient is 0.9997 , which is significantly better than other methods. In 2019, William T. Hrinivich [18] et al. reconstructed the reconstruction of ring-shaped applicators and oval applicators in MRI-guided cervical cancer through a model-to-image registration algorithm, proving that the accuracy and time of the algorithm fully meet the clinical needs and make treatment The automation of the plan is a step forward. Hyunuk Jung [19] [20] et al. completed the segmentation of the applicator in high-dose cervical cancer by constructing a U-net network, and then generated the trajectory of the applicator through the voxel clustering algorithm to complete the reconstruction of the applicator, and The model was evaluated using Hausdorff distance, Days similarity coefficient, and the average difference in needle tip positions. On the basis of this work, the segmentation and reconstruction of the interpolation needle in brachytherapy for cervical cancer were also studied by the same algorithm. Experiments have proved that the reconstruction of interpolation needles and applicators in brachytherapy for cervical cancer is of great help to the clinic. Paolo Zaffino [21] et al. proposed an algorithm based on Convolutional Neural Network (CNN) in 2019 for fully automatic segmentation of multiple closely spaced applicators in nuclear magnetic images. The average error distance of the final segmentation is $2.0 \pm 3.4 \mathrm{~mm}$, the proportion of false positive and false negative applicators were $6.7 \%$ and $1.5 \%$, respectively. By combining Attention gate and U-net in 2020, Xianjin Dai [22] et al. used a total variation (TV) regularization to construct a model to complete the detection and reconstruction of interpolation needles in brachytherapy for prostate cancer with high dose rate MRI, and evaluate the model through tip deviation and movement deviation. Yupei Zhang [23] et al. studied the reconstruction of the position of interpolation needles in prostate cancer guided by ultrasound images through the combination of U-net network and attention in 2020. Similar to the work of Xianjin Dai, they are all interpolation needles for brachytherapy of prostate cancer. For positioning and reconstruction, the only difference is that the ultrasound and MRI guided images are reconstructed separately, and the reconstruction results are good enough to meet the clinical needs. Fuyue Wang [24] et al. also applied the automatic segmentation of interpolation needles in brachytherapy of prostate cancer by constructing U-net network in 2020 , and proved that the model can accurately reconstruct the trajectory of interpolation needles.

In the past two or three years, great attention has been paid to target area delineation and applicator reconstruction. Different network models have also 
been constructed for reconstruction of applicators or interpolation pins. However, the change is inseparable, basically based on the idea of deep learning, using U-net network or its variants to reconstruct or segment the applicator. Target area sketching is also done using supervised neural networks.

\section{Dose Calculation}

Deep learning has not done much research on dose calculation. It is still relatively blank at this stage, but dose calculation is an important step in brachytherapy, and further research is needed [25].

Marc Morcos [26] et al. calculated and studied the dose of nuclear magnetically guided brachytherapy for cervical cancer based on Monte Carlo in 2020, and evaluated the effect of different rotation angles on the intensity-modulated radiotherapy on the dose, laid the foundation for the treatment of complex cervical cancer by intensity modulated radiotherapy. In 2020, Ximeng Mao [27] et al. built a fast brachytherapy deep learning model through convolutional neural networks for dose calculation in brachytherapy planning. The results show that the accuracy is similar to the results obtained by the Monte Carlo algorithm, but the calculation speed is much faster and can be extended to other tumor sites.

\section{Application of Treatment Planning System}

The treatment plan is formulated by the physicist by the treatment planning system. Many semi-automatic or fully automatic treatment planning systems have been developed to improve the quality of treatment planning while reducing planning time. Some of them have been integrated and successfully tested in commerce. Therefore, deep learning methods are also suitable for the automation of treatment planning [6]. In 2011, Timmy Siauw [28] et al. proposed an optimization model and fast heuristic algorithm for calculating HDR brachytherapy dose planning, inverse planning (IPIP), and evaluated the measured dose and compared it with the standard dose. Studies have shown that the prostate dose obtained by the algorithm used in this study has clinical significance. C Guthier [29] et al. studied a reverse planning for low-dose-rate brachytherapy in 2015, applied the idea of compressed sensing, developed a reverse planning algorithm, and optimized it to adapt this algorithm to the current best reverse Algorithms are compared and faster. The algorithm can also effectively reduce the cost of intervention. Alexandru Nicolae [30] et al. 2016 used machine learning algorithms to automatically generate a low dose rate brachytherapy plan for the prostate, and compared the pre- and post-implantation treatment plans generated by the machine learning algorithm with the plans made by the physicist. The results showed that the machine-generated plans quality was same with the plan made by the physicist, but it can reduce the planning time and resources. In 2019, Chenyang Shen [31] et al. developed a weight adjustment strategy network based on reverse optimization after high-dose-rate cervical cancer in order to observe the planned dose volume histogram and adjust the organ weighting fac- 
tor in real time. The experimental results prove that the quality is improved by $10.7 \%$ compared to the plan made by the physicist. Maryam Golshan [32] et al. built a model through convolutional neural networks in 2019 to complete the automatic detection of seeds in brachytherapy under the guidance of three-dimensional ultrasound images. The results show that the estimated time for each needle is 1 minute, and the total time is less than 15 minutes. Compared with manual, the model obtained higher accuracy. By 2020, Alexandru Nicolae [33] et al. randomly compared the treatment plan based on machine learning with the traditional manual treatment plan to evaluate the total planning time between the two groups and the dose measurement results after 30 days of implantation, compared with the traditional Manual planning has a great advantage in the time of treatment planning based on machine learning.

\section{Others}

In addition to the application of deep learning technology in the above aspects [34], some scholars have also conducted a series of studies in toxicity prediction and other aspects. Several research centers have confirmed the value of machine learning methods in prediction, and now have used deep learning to study the toxicity of lungs, prostate, etc. In 2017, Xin Zhen [34] et al. study on the prediction of rectal toxicity in cervical cancer radiotherapy based on transfer learning deep convolutional neural network. Convolutional neural network was used to analyze rectal dose distribution and predict rectal toxicity. 42 patients were collected and overcome by transfer learning for quantity problems, training on VGG-16, fine-tuning of the patient's rectal display dose map, and comparison with traditional dose volume parameters, studies have shown that pre-trained $\mathrm{CNN}$ can simulate rectal dose distribution and predict rectal toxicity after cervical cancer radiotherapy. There are also some studies on the prediction of the survival rate of deep learning in the next 5 years.

In terms of toxicity research, the application of deep learning is not so extensive for the time being. Future research areas in this area have better prospects. Of course, there are other areas worthy of improvement.

\section{Conclusions}

From the above, we can see that deep learning is becoming more and more widely used in brachytherapy, especially in the treatment of cervical cancer and prostate cancer has been widely developed. Often focusing on target area delineation, applicator reconstruction, radiotherapy planning system and dose calculation, etc., it provides new assistance for improving the clinical treatment effect and the automation of the treatment planning system.

The automation of the brachytherapy plan is one of the future development directions. It is worthwhile to conduct a series of studies to construct different networks through deep learning to solve the problems in brachytherapy. At this stage, target area delineation and applicator reconstruction are both moving in 
the direction of automation. Future dose calculation and dose optimization are also worthy of attention.

\section{Conflicts of Interest}

The authors declare no conflicts of interest regarding the publication of this paper.

\section{References}

[1] 李龙婕, 邓晓琴. 宫颈癌近距离放射治疗进展 [J]. 大连医科大学学报, 2019, 41(3): 193-198.

[2] 王金花, 宋金维, 王建东. 人工智能在宫颈癌笁查中的研究进展 [J]. 癌症进展, 2019, 17(13): 1503-1505.

[3] Lee, J.H., Ha, E.J. and Kim, J.H. (2019) Application of Deep Learning to the Diagnosis of Cervical Lymph Node Metastasis from Thyroid Cancer with CT. European Radiology, 29, 5452-5457. https://doi.org/10.1007/s00330-019-06098-8

[4] Doyle, L.A., Yondorf, M., Peng, C., Harrison, A.S. and Den, R.B. (2018) Process Mapping and Time Study to Improve Efficiency of New Procedure Implementation for High-Dose Rate Prostate Brachytherapy. Journal of Healthcare Quality, 40, 19-26.

[5] Meyer, P., Noblet, V., Mazzara, C. and Lallement, A. (2018) Survey on Deep Learning for Radiotherapy. Computers in Biology and Medicine, 98, 126-146.

https://doi.org/10.1016/j.compbiomed.2018.05.018

[6] William, W., Ware, A., Basaza-Ejiri, A.H. and Obungoloch, J. (2018) A Review of Image Analysis and Machine Learning Techniques for Automated Cervical Cancer Screening from Pap-Smear Images. Computer Methods and Programs in Biomedicine, 164, 15-22.

[7] Chen, J., Remulla, D., Nguyen, J.H., Aastha, D., Liu, Y., Dasgupta, P., Hung, A.J. (2019) Current Status of Artificial intelligence Applications in Urology and Their Potential to Influence Clinical Practice. BJU International, 124, 567-577. https://doi.org/10.1111/bju.14852

[8] Cunha, J.A.M., Flynn, R., Bélanger, C., et al. (2020) Brachytherapy Future Directions. Seminars in Radiation Oncology, 30, 94-106. https://doi.org/10.1016/j.semradonc.2019.09.001

[9] Tajbakhsh, N., Jeyaseelan, L., Li, Q., Chiang, J.N., Wu, Z.H. and Ding, X.W. (2020) Embracing Imperfect Datasets: A Review of Deep Learning Solutions for Medical Image Segmentation. Medical Image Analysis, 63, Article ID: 101693. https://doi.org/10.1016/j.media.2020.101693

[10] Allman, D., Reiter, A. and Bell, M.A.L. (2018) Photoacoustic Source Detection and Reflection Artifact Removal Enabled by Deep Learning. IEEE Transactions on Medical Imaging, 37, 1464-1477.

[11] Huang, X., Wang, J., Tang, F., Zhong, T. and Zhang, Y. (2018) Metal Artifact Reduction on Cervical CT Images by Deep Residual Learning. BioMedical Engineering Online, 17, Article No. 175. https://doi.org/10.1186/s12938-018-0609-y

[12] Karimi, D., Zeng, Q., Mathur, P., et al. (2019) Accurate and Robust Deep Learning-Based Segmentation of the Prostate Clinical Target Volume in Ultrasound Images. Medical Image Analysis, 57, 186-196.

https://doi.org/10.1016/j.media.2019.07.005 
[13] Lei, Y., Tian, S., He, X., et al. (2019) Ultrasound Prostate Segmentation Based on Multidirectional Deeply Supervised V-Net. Medical Physics, 46, 3194-3206. https://doi.org/10.1002/mp.13577

[14] Orlando, N., Gillies, D.J, Gyacskov, I., Romagnoli, C., D’Souza, D. and Fenster, A. (2020) Automatic Prostate Segmentation Using Deep Learning on Clinically Diverse 3D Transrectal Ultrasound Images. Medical Physics, 47, 2413-2426. https://doi.org/10.1002/mp.14134

[15] 秦楠楠, 薛旭东, 吴爱林, 等. 基于 U-net 卷积神经网络的宫颈癌临床靶区和危 及器官自动勾画的研究 [J]. 中国医学物理学杂志, 2020, 37(4): 524-528.

[16] Gessert, N., Priegnitz, T., Saathoff, T., et al. (2019) Spatio-Temporal Deep Learning Models for Tip force Estimation during Needle Insertion. International Journal of Computer Assisted Radiology and Surgery, 14, 1485-1493. https://doi.org/10.1007/s11548-019-02006-Z

[17] Hrinivich, W.T., Morcos, M., Viswanathan, A. and Lee, J.H. (2019) Automatic Tandem and Ring Reconstruction Using MRI for Cervical Cancer Brachytherapy. Medical Physics, 46, 4324-4332. https://doi.org/10.1002/mp.13730

[18] Jung, H., Shen, C.Y., Gonzalez, Y., Albuquerque, K. and Jia, X. (2019) Deep-Learning Assisted Automatic Digitization of Interstitial Needles in 3D CT Image Based High Dose-Rate Brachytherapy of Gynecological Cancer. Physics in Medicine \& Biology, 64, Article ID: 215003. https://doi.org/10.1088/1361-6560/ab3fcb

[19] Jung, H., Gonzalez, Y., Shen, C., Klages, P. and Albuquerque, K. (2019) Deep Learning Assisted Automatic Digitization of Applicators in 3D CT Image-Based High-Dose-Rate Brachytherapy of Gynecological Cancer. Brachytherapy, 18, 841-851. https://doi.org/10.1016/j.brachy.2019.06.003

[20] Zaffino, P., Pernelle, G., Mastmeyer, A., et al. (2019) Fully Automatic Catheter Segmentation in MRI with 3D Convolutional Neural Networks: Application to MRI-Guided Gynecologic Brachytherapy. Physics in Medicine \& Biology, 64, Article ID: 165008. https://doi.org/10.1088/1361-6560/ab2f47

[21] Dai, X., Lei, Y., Zhang, Y., et al. (2020) Automatic Multi-Catheter Detection Using Deeply Supervised Convolutional Neural Network in MRI-Guided HDR Prostate Brachytherapy. Medical Physics. https://doi.org/10.1002/mp.14307

[22] Zhang, Y., Lei, Y., Qiu, R.L.J., et al. (2020) Multi-Needle Localization with Attention U-Net in US-Guided HDR Prostate Brachytherapy. Medical Physics.

[23] Wang, F., Xing, L., Bagshaw, H., Buyyounouski, M. and Han, B. (2020) Deep Learning Applications in Automatic Needle Segmentation in Ultrasound-Guided Prostate Brachytherapy. Medical Physics. https://doi.org/10.1002/mp.14328

[24] Wang, X., Wang, P., Li, C., et al. (2018) An Automated Dose Verification Software for Brachytherapy. Journal of Contemporary Brachytherapy, 10, 478-482. https://doi.org/10.5114/jcb.2018.79396

[25] Morcos, M. and Enger, S.A. (2020) Monte Carlo Dosimetry Study of Novel Rotating MRI-Compatible Shielded Tandems for Intensity Modulated Cervix Brachytherapy. European Journal of Medical Physics, 71, 178-184. https://doi.org/10.1016/j.ejmp.2020.02.014

[26] Mao, X.M., Pineau, J., Keyes, R. and Enger, S.A. (2020) RapidBrachyDL: Rapid Radiation Dose Calculations in Brachytherapy via Deep Learning. International Journal of Radiation Oncology, Biology, Physics.

[27] Siauw, T., Cunha, A., Atamtürk, A., Hsu, I.-C., Pouliot, J. and Goldberg, K. (2011) IPIP: A New Approach to Inverse Planning for HDR Brachytherapy by Directly Op- 
timizing Dosimetric Indices. Medical Physics, 38, 4045-4051.

https://doi.org/10.1118/1.3598437

[28] Guthier, C., Aschenbrenner, K.P., Buergy, D., et al. (2015) A New Optimization Method Using a Compressed Sensing Inspired Solver for Real-Time LDR-Brachytherapy Treatment Planning. Physics in Medicine and Biology, 60, 2179-2194. https://doi.org/10.1088/0031-9155/60/6/2179

[29] Nicolae, A., Morton, G., Chung, H., et al. (2017) Evaluation of a Machine-Learning Algorithm for Treatment Planning in Prostate Low-Dose-Rate Brachytherapy. International Journal of Radiation Oncology, Biology, Physics, 97, 822-829. https://doi.org/10.1016/j.ijrobp.2016.11.036

[30] Shen, C., Gonzalez, Y., Klages, P., et al. (2019) Intelligent Inverse Treatment Planning via Deep Reinforcement Learning, a Proof-of-Principle Study in High Dose-Rate Brachytherapy for Cervical Cancer. Physics in Medicine \& Biology, 64, Article ID: 115013. https://doi.org/10.1088/1361-6560/ab18bf

[31] Golshan, M., Karimi, D., Mahdavi, S., et al. (2020) Automatic Detection of Brachytherapy Seeds in 3D Ultrasound Images Using a Convolutional Neural Network. Physics in Medicine \& Biology, 65, Article ID: 35016. https://doi.org/10.1088/1361-6560/ab64b5

[32] Nicolae, A., Semple, M., Lu, L., et al. (2020) Conventional vs. Machine Learning-Based Treatment Planning in Prostate Brachytherapy: Results of a Phase I Randomized Controlled Trial. Brachytherapy, 19, 470-476. https://doi.org/10.1016/j.brachy.2020.03.004

[33] Tian, Z., Yen, A., Zhou, Z., et al. (2019) A Machine-Learning-Based Prediction Model of Fistula Formation after Interstitial Brachytherapy for Locally Advanced Gynecological Malignancies. Brachytherapy, 18, 530-538.

https://doi.org/10.1016/j.brachy.2019.04.004

[34] Zhen, X., Chen, J.W., Zhong, Z.C., Hrycushko, B., Zhou, L.H., Jiang, S., Albuquerque, K. and Gu, X.J. (2017) Deep Convolutional Neural Network with Transfer Learning for Rectum Toxicity Prediction in Cervical Cancer Radiotherapy: A Feasibility Study. Physics in Medicine \& Biology, 62, 8246-8263. 